\title{
DIFFERENTIATION OF DICHOTOMOUS EMOTIONAL STATES IN ELECTRODERMAL ACTIVITY SIGNALS USING HIGHER-ORDER CROSSING FEATURES AND PARAMETRIC CLASSIFIERS
}

\author{
Yedukondala Rao Veeranki ${ }^{1}$, Nagarajan Ganapathy ${ }^{1,2}$, Ramakrishnan Swaminathan ${ }^{1}$ \\ ${ }^{1}$ Biomedical Engineering Group, Department of Applied Mechanics, Indian Institute of Technology \\ Madras, Chennai, Tamil Nadu, India - 600036 \\ ${ }^{2}$ PLRI for Medical Informatics of TU Braunschweig and Hannover Medical School, Germany
}

\author{
Corresponding Author: Yedukonddala Rao Veeranki, \\ Email: ykraoveeranki@gmail.com \\ Tel: (+91)-44 22575065 \\ https://doi.org/10.34107/BiomedSciInstrum.57.04322
}

\begin{abstract}
Prediction and recognition of happy and sad emotional states play important roles in many aspects of human life. In this work, an attempt has been made to classify them using Electrodermal Activity (EDA). For this, EDA signals are obtained from a public database and decomposed into tonic and phasic components. Features, namely Hjorth and higher-order crossing, are extracted from the phasic component of the signal. Further, these extracted features are fed to four parametric classifiers, namely, linear discriminant analysis, logistic regression, multilayer perceptron, and naive bayes for the classification. The results show that the proposed approach can classify the dichotomous happy and sad emotional states. The multilayer perceptron classifier is accurate $(85.7 \%)$ in classifying happy and sad emotional states. The proposed method is robust in handling the dynamic variation of EDA signals for happy and sad emotional states. Thus, it appears that the proposed method could be able to understand the neurological, psychiatrical, and biobehavioural mechanisms of happy and sad emotional states.
\end{abstract}

Keywords: Electrodermal activity, Happy, Sad, Higher-order crossing, Hjorth, and Parametric classifiers

\section{INTRODUCTION}

Emotions are mental states or feelings associated with a nervous system that are related to physiological changes, subjective feelings, cognitive processes, and behavioral responses [1]. Ekman posits six universally recognized basic emotions, namely anger, disgust, fear, happiness, sadness, and surprise [2], [3]. Among the six basic emotions, happy and sad emotions are frequently experienced by humans, which is also called a core affect [3], [4]. Happiness and sadness are dichotomous since any degree of happiness preclude sadness, and, conversely, any degree of sadness precludes happiness [5]. Happiness is a positive state associated with various progressive outcomes, including increased social behavior and problem-solving capability [6], [7]. Sadness is an adverse mental condition related to solitude, depression, grief, anxiety, and distress [8]. The detection and classification of happy and sad emotional states can be useful for understanding mental health, prediction of major depressive disorder, and the treatment of emotion disorders [8].

The emotional states are characterized using Russells circumplex model [9]. The model captures the similarities and differences between affective states along arousal and valence dimensions. The valence expresses the amount of negative and positive felt by the subject, and arousal characterizes the emotional intensity. Happiness and sadness can be described by similar arousal levels but opposing valence [9], [10].

The emotions can be recognized by both physiological and non-physiological trait-based approaches [11]. Emotion recognition based on physiological trait-based approaches has attracted more attention since the measurements can be quantified and cannot be easily controlled. The physiological trait-based 